\title{
COMUNICANDO DATOS MASIVOS DEL SECTOR PUBBLICO LOCAL EN REDES SOCIALES. ANÁLISIS DE SENTIMIENTO EN TWITTER
}

\author{
Local public sector big data communication on social \\ media. A sentiment analysis in Twitter
}

\section{J. Ignacio Criado y Julián Villodre}

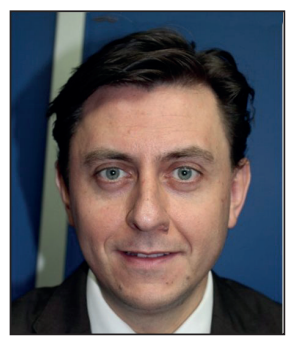

J. Ignacio Criado es profesor en el Departamento de Ciencia Política y Relaciones Internacionales de la Universidad Autónoma de Madrid y research fellow del Center for Technology in Government, State University of New York (SUNY at Albany). Ha sido visiting fellow en el Oxford Internet Institute. Director de NovaGob Lab y cofundador de la red social NovaGob. Ha recibido varios reconocimientos a su investigación, entre ellos, el Premio Julián Marías 2016 de la Comunidad de Madrid.

https://orcid.org/0000-0002-9184-9696

ignacio.criado@uam.es

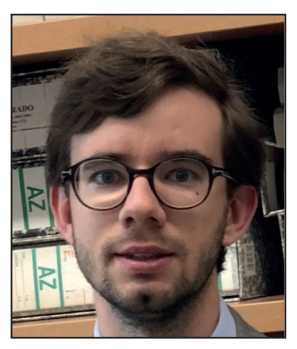

Julián Villodre es investigador contratado FPI-UAM y candidato a doctor en el Departamento de Ciencia Política y Relaciones Internacionales de la Universidad Autónoma de Madrid (UAM). Graduado en Ciencia Política y Administración Pública y master en Democracia y Gobierno (ambos por la UAM), colabora como investigador en el NovaGob Lab y en la red social NovaGob. https://orcid.org/0000-0003-0468-8802

julian.villodrede@uam.es

Universidad Autónoma de Madrid, Departamento de Ciencia Política y Relaciones Internacionales Ciudad Universitaria de Cantoblanco Marie Curie, 1. 28049 Madrid, España

\section{Resumen}

La rápida difusión de las redes sociales en las administraciones públicas requiere un análisis desde la información y comunicación institucional. Uno de los aspectos detectados recientemente es el tono con el que emiten sus mensajes las organizaciones públicas, a través del cual pueden fomentar la colaboración y participación ciudadana. Este trabajo analiza el tono que los ayuntamientos españoles de más de 50.000 habitantes están empleando en sus perfiles de redes sociales. Se parte de dos preguntas de investigación: (a) cuál es el tono general empleado por los ayuntamientos en su uso de redes sociales; y (b) cuáles son los factores que explican la elección de un tono u otro. Recurriendo al estudio de Twitter mediante técnicas de big data y análisis estadísticos, el artículo encuentra un tono comunicativo general positivo. Los factores organizativos, institucionales y ambientales clásicos parecen no tener una influencia estadísticamente significativa sobre el tono. El artículo concluye con propuestas de mejora de cara a futuros estudios en diferentes aspectos a partir de los resultados obtenidos.

\section{Palabras clave}

Administración pública; Administración local; Ayuntamientos; Gobierno digital; Información pública; Comunicación política; Redes sociales; Medios sociales; Datos masivos; Análisis de sentimiento; Twitter; España.

\section{Abstract}

The rapid adoption of social media by public administrations deserves attention from the side of institutional information and communication. One of the points recently identified is the tone used by public sector organizations to deliver their messages, as it may encourage citizen collaboration and participation. This paper explores the tone that Spanish city councils with more than 50,000 inhabitants are using in their social media profiles. This paper is based in two research questions: (a) What is the general tone used by the city councils in their use of social media?; (b) What explanatory factors are behind the adoption of one tone or another? Studying Twitter through big data techniques and statistical analysis, this study finds a positive general communicative tone. Traditional organizational, institutional and environmental factors do not seem to have a statistical significant influence over the tone. This article concludes with proposals for improvement on future research derived from the research results. 


\section{Keywords}

Public administrations; Local governments; City councils; Digital government; Public information; Political communication; Social networks; Social media; Big data; Sentiment analysis; Twitter; Spain.

Criado, J. Ignacio; Villodre, Julián (2018). “Comunicando datos masivos del sector público local en redes sociales. Análisis de sentimiento en Twitter". El profesional de la información, v. 27, n. 3, pp. 614-623.

https://doi.org/10.3145/epi.2018.may.14

\section{Introducción}

La extensión del uso de redes sociales en el sector público junto a su potencial altamente disruptivo (Mergel, 2016; Criado; Sandoval-Almazán; Gil-García, 2013), ha generado grandes expectativas sobre la capacidad de las tecnologías sociales para mejorar las áreas de transparencia, colaboración y participación (Bertot; Jaeger; Grimes, 2010). A medida que aumenta la popularidad de estas plataformas se hace más necesario entender cómo se puede fomentar con ellas la interacción entre ciudadanía y las administraciones públicas (Mergel, 2013).

Este trabajo se centra en uno de los elementos recientemente identificados por la bibliografía como clave para el fomento de la comunicación y participación en redes sociales: el tono. En concreto este artículo pretende conocer los determinantes del tono (positivo, negativo o neutro) de la comunicación en redes sociales de las administraciones locales españolas, teniendo en cuenta diferentes dimensiones.

Metodológicamente se ofrece una perspectiva plural que integra diversas técnicas de recogida y análisis de datos que facilitan su triangulación. Por un lado, se sitúa en el nivel local de gobierno y se propone explorar el tono (negativo, neutral, positivo) con el que los ayuntamientos comunican datos e información a través de su perfil social en Twitter mediante empleo de técnicas de datos masivos (big data) (Arcila-Calderón; Barbosa-Caro; Cabezuelo-Lorenzo, 2016). El trabajo recurre además al análisis estadístico con los datos de una encuesta realizada a los gestores públicos de redes sociales en los municipios españoles de más de 50.000 habitantes, un grupo esencial de organizaciones públicas analizado en otros estudios previos sobre información pública y comunicación a través de portales web (Fernández-Falero et al., 2017; Manfredi-Sánchez; Corcoy-Rius; Herranz-dela-Casa, 2017). Por su parte, las dimensiones y factores seleccionados en el marco analítico se encuadran dentro de las dimensiones organizativas, institucionales y ambientales propuestas desde el marco de medición del éxito de gobierno electrónico Enacting electronic government success (EEGS) de Gil-García (2012), recientemente utilizado por Criado, Rojas-Martín y Gil-García (2017) en el ámbito de las redes sociales en el sector público.

La dimensión de los datos masivos tiene un carácter fundamental en nuestro trabajo. De forma general, su importancia para los estudios de sector público radica en su potencial para obtener datos y dar sentido a los mismos en términos de escucha activa, gestión de sistemas de conocimiento, análisis de opinión y gestión de estrategias de comunicación
(Criado; Villodre, 2018; Eom; Hwang; Kim, 2017; Loukis; Charalabidis; Androutsopoulou, 2017). En particular, este estudio emplea tecnologías big data como mecanismo de extracción de más de 12.000 tweets de municipios españoles y como estrategia analítica a través de la computación de estos datos con análisis de sentimientos. Estas cifras serían impensables para una descarga y codificación manual, y aún cuando puedan ser consideradas insuficientes en términos de grandes datos, una consideración de los big data únicamente en torno al tamaño o volumen de su información es insuficiente (Arcila-Calderón; Barbosa-Caro; Cabezuelo-Lorenzo, 2016) si no va acompañada de técnicas indispensables para computar esa información, tenga el volumen que tenga, en tiempo razonable. Entre ellas, también se beneficia nuestro estudio del empleo de análisis de sentimiento.

Por su carácter reciente, el análisis de sentimiento en el sector público cuenta con amplio espacio para su desarrollo. En los últimos años destaca el trabajo de Zavattaro, French y Mohanty (2015), cuyos análisis sobre entidades locales estadounidenses muestran una posible relación entre el tono neutral de los tweets y la estrategia gubernamental de mera provisión de información. En este mismo sentido el estudio de Hofmann et al. (2013) relaciona el empleo de sentimiento positivo en las comunicaciones de agencias públicas a través de Facebook con una exitosa implementación de las estrategias de comunicación en redes sociales dentro del sector público.

Sin embargo ninguno de estos trabajos ha explorado los factores explicativos que llevan a las administraciones públicas a la adopción de unos sentimientos $u$ otros en los procesos de comunicación con la ciudadanía. Este trabajo retoma la necesidad planteada por Zavattaro, French y Mohanty (2015) de continuar con estos análisis bajo otros contextos, y se propone examinar el sentimiento general de los tweets en los perfiles sociales de los municipios españoles con más de 50.000 habitantes, tratando además de identificar factores clave para el fomento del tono empleado. Este artículo plantea las siguientes preguntas de investigación:

P1. ¿Cuál es el tono general empleado por los ayuntamientos en su uso de redes sociales digitales?

P2. ¿Qué factores explicativos se encuentran detrás de la adopción de un tono u otro?

Para acometer este propósito el artículo presenta primero una revisión bibliográfica, marco teórico-analítico, y formulación de hipótesis; luego se detiene en el diseño y metodología de la investigación, para después mostrar los resultados y las conclusiones. 


\section{Revisión bibliográfica, marco teórico e hipótesis}

En general la bibliografía sobre comunicación, engagement y fomento de la participación a través de redes sociales se ha movido bajo la búsqueda del ideal de ciudadano activo. Este es el resultado de un proceso de empoderamiento que lo iguala como "compañero" del propio gobierno en el proceso de decisión pública (Bryer, 2013). Esa relación genera una serie de sinergias que refuerzan la confianza y la satisfacción con las instituciones (Lev-On; Steinfeld, 2015). Sin embargo se conoce poco sobre los mecanismos que hay detrás de las nuevas dinámicas de interacción.

A modo de ejemplo, el tono general encontrado en los análisis sobre perfiles de organizaciones públicas por los estudios más recientes ha sido el neutro. Este tipo de sentimientos se han venido relacionando con estrategias de provisión de información dentro del sector público (Zavattaro; French; Mohanty, 2015; Mergel, 2013; Sixto-García, 2012), mientras que un tono positivo ha tendido a ofrecer en los perfiles sociales de las administraciones públicas una respuesta más animada del ciudadano, impulsando la participación y la colaboración entre la administración y la comunidad que poco a poco va creando (Zavattaro; French; Mohanty, 2015), que sin embargo todavía está lejos de generalizarse.

Como observa Sixto-García (2012), a pesar de que las organizaciones públicas otorgan gran importancia a las redes sociales digitales para comunicarse con los usuarios, continúan usando fórmulas unidireccionales en su comunicación (como prácticas comunicativas para difundir eventos fundadas en el potencial viral de estas plataformas). Por ello en relación a la $\mathrm{P} 1$ sostenemos que:

H1. Los gestores de redes sociales de las organizaciones públicas emplearán un tono generalmente neutro, en línea con una estrategia de provisión de información institucional.

Este trabajo se propone también detectar variables que afectan de una forma u otra al tono con el que las administraciones emiten sus mensajes a través de tecnologías sociales. Para ello, y en respuesta a nuestra P2 sobre los factores explicativos que hay detrás de la adopción de un tono u otro, se recurre al citado modelo EEGS, ya utilizado con anterioridad en estudios sobre perfiles sociales (Criado; Rojas-Martín; Gil-García, 2017).
El modelo EEGS se sitúa en un conjunto de marcos teóricos de reciente creación que intentan dar respuesta a los problemas específicos del gobierno digital. En un momento de adopción masiva de tecnologías por parte del sector público, el EEGS enfrenta la cuestión fundamental de cómo medir de forma sistemática e integral esas nuevas aplicaciones (Gil-García, 2012). En base al marco teórico de Fountain (2001), heredero del institucionalismo y que pone el acento en la importancia del impacto del entorno y de las instituciones en la propia organización, Gil-García (2012) organiza su modelo en tres grandes grupos de factores:

- estructuras organizativas;

- condicionantes institucionales;

- factores ambientales.

La tabla 1 introduce la relación entre estas dimensiones, y las variables independientes que componen nuestras principales hipótesis.

Por su carácter reciente, el análisis de sentimiento en el sector público cuenta con amplio espacio para su desarrollo

Las variables que se presentan en la tabla 1 responden a la medición de un elemento particular del éxito. Como ya hemos destacado con anterioridad, el tono de los ayuntamientos en sus perfiles sociales puede denotar el avance hacia una estrategia positiva o negativa de adopción de redes sociales (Zavattaro; French; Mohanty, 2015). Un tono positivo mostrará una estrategia más exitosa dentro de una organización pública que otra que emplee un tono más neutro, y que por tanto se centre en la mera provisión de información y no en alentar la participación y la colaboración (Zavattaro; French; Mohanty, 2015).

La primera dimensión que muestra la tabla 1 hace referencia a las estructuras organizativas, y se centra tanto en la formación recibida por los gestores en redes sociales como en las unidades y departamentos que gestionan los perfiles sociales. La formación recibida por los miembros de la organización se ha venido considerando como un elemento fundamental a la hora de aproximarnos al uso y gestión de

Tabla 1. Dimensiones del EEGS y variables seleccionadas

\begin{tabular}{|l|l|l|}
\hline \multicolumn{1}{|c|}{ Dimensión } & \multicolumn{1}{c|}{ Descripción de la dimensión } & \multicolumn{1}{c|}{ Variables seleccionadas } \\
\hline $\begin{array}{l}\text { Estructuras } \\
\text { organizativas }\end{array}$ & $\begin{array}{l}\text { Se refiere a las características propias de la organización, como el número de gente } \\
\text { trabajando en ella, el porcentaje de fondos dedicados a las tecnologías digitales, la } \\
\text { formación en estas tecnologías o las divisiones de la agencia. }\end{array}$ & $\begin{array}{l}\text { Formación recibida en redes sociales, } \\
\text { unidades que gestionan los perfiles } \\
\text { sociales. }\end{array}$ \\
\hline $\begin{array}{l}\text { Condicionantes } \\
\text { institucionales }\end{array}$ & $\begin{array}{l}\text { Se refiere a todas aquellas regulaciones, planes de medios sociales y normas } \\
\text { (formales o informales) que afectan tanto a la tecnología seleccionada, como a los } \\
\text { resultados de su implementación en la organización. }\end{array}$ & $\begin{array}{l}\text { Existencia de planes de medios socia- } \\
\text { lescepción de la cultura organiza- } \\
\text { tiva como inhibidor. }\end{array}$ \\
\hline $\begin{array}{l}\text { Factores } \\
\text { ambientales }\end{array}$ & $\begin{array}{l}\text { Se refiere a una gran cantidad de factores externos que afectan a la implementa- } \\
\text { ción en la organización. }\end{array}$ & $\begin{array}{l}\text { Tamaño del municipio (población), } \\
\text { edad media de la población del } \\
\text { municipio. }\end{array}$ \\
\hline
\end{tabular}

Fuente: elaborado a partir de Gil-García (2012). 
redes sociales: en la medida en que los gestores conocen los potenciales de las tecnologías sociales, son capaces de superar algunas de las barreras en su uso (Ma, 2016).

Por ello consideramos la siguiente H2: Las organizaciones públicas tenderán a emplear un tono más positivo en las redes sociales digitales si sus gestores han recibido formación en redes sociales digitales.

Por otro lado, los departamentos que gestionan estos perfiles también ofrecen apuntes de interés debido a su importancia a la hora de medir la institucionalización de tecnologías en el sector público (Mergel, 2016), y su relación con prácticas centralizadas o descentralizadas de implementación de estrategias. Los departamentos a cargo de la gestión de perfiles sociales se han ubicado generalmente en áreas de comunicación e informática (Criado; Rojas-Martín, 2013; 2015), normalmente más preocupados por la provisión de información (Zavattaro; French; Mohanty, 2015), si bien se destaca de la misma forma la intensidad con la que a menudo los departamentos trabajan conjuntamente para la gestión de estos perfiles (Criado; Rojas-Martín, 2015).

Por ello la H3 es: Las organizaciones públicas tenderán a usar un tono más positivo en las redes sociales digitales en la medida que su gestión se encuentre descentralizada.

La segunda dimensión identificada según la tabla 1 son los condicionantes institucionales: guías, regulaciones y normas, formales e informales, que se entrelazan en la adopción y uso de las tecnologías. El empleo de planes de medios sociales en el sector público está relacionado directamente con elementos de políticas públicas (Chen et al., 2016). Estos planes guían las estrategias para superar desviaciones y fallas en el control y en los usos de estas tecnologías, además de ayudar en la monitorización y en la sistematización paso a paso de las estrategias concretas (Chen et al., 2016). Son documentos formales que reúnen los puntos más relevantes para la gestión adecuada de los medios sociales en las administraciones públicas, buscando formalizar, organizar y orientar. La ausencia de estos planes de medios sociales en una organización no implica directamente una implementación inadecuada, pero las instituciones que las han elaborado demuestran su compromiso con estas estrategias.

Así, nuestra $\mathrm{H} 4$ es: Las organizaciones públicas tenderán a usar un tono más positivo en las redes sociales digitales en la medida que dispongan de planes de medios sociales que orienten, formalicen y organicen la gestión de sus perfiles.

Los elementos relacionados con la cultura organizativa también se han venido considerando como relevantes. Esto es así por su tratamiento como inhibidora de cualquier elemento de innovación dentro de la organización (Bailey; Singleton, 2010), y una de las tres barreras de entrada más importantes en la implementación de tecnologías sociales dentro de la organización. Una percepción de esa cultura como obstaculizadora podría derivar en la mera consideración de los perfiles como espacios informativos. Por ello:

H5. Las organizaciones públicas tenderán a emplear un tono más positivo en las redes sociales digitales si la cultura organizativa no se percibe como un inhibidor en la adopción de tecnologías sociales.
Finalmente la última dimensión que muestra la tabla 1 se relaciona con un grupo de gran heterogeneidad, referido a los aspectos socio-demográficos, políticos y económicos que rodean a la organización. De estos factores ambientales por limitaciones de espacio el artículo se centra en su vertiente más socio-demográfica, seleccionando el tamaño del municipio y la edad media de sus habitantes como variables relevantes. Por un lado la edad es uno de los elementos clave en cualquier estudio sobre esta materia, de manera que en el uso de tecnologías sociales, el estudio de la edad se ha relacionado directamente con la existencia de brecha digital (Tello, 2008). Podemos sostener que:

H6. Las organizaciones públicas tenderán a emplear un tono más positivo en las redes sociales digitales en la medida que la población del territorio en el que se enmarcan sea más joven.

El tamaño del municipio es otra variable clásica que ha demostrado una relevancia importante a la hora de observar las diferencias en la gestión de los servicios y la implementación de políticas públicas. Por su actualidad conviene destacar un estudio sobre administración electrónica realizado por el Gobierno de Aragón (OASI, 2016), que daba algunos datos interesantes:

- los municipios de mayor tamaño disponían de perfiles "oficiales" (asumiendo con ello su carácter de página oficial, y no de perfil personal) en mayor número de redes sociales ( $85 \%$ de los casos), mientras que los de menor tamaño en población se decantaban preferentemente por abrir un perfil sólo en Facebook;

- los de mayor tamaño en población disponían en mayor medida de planes estratégicos, y eran capaces de cumplir de forma más efectiva sus compromisos y competencias en materias de participación y provisión de información que los municipios de menor tamaño.

Por ello se puede pensar que:

H7. Las organizaciones públicas tenderán a emplear un tono más positivo en las redes sociales digitales cuanto mayor sea el tamaño del municipio.

\section{Metodología y operacionalización de variables}

Esta investigación se propone explorar el tono general con el que se expresan los ayuntamientos españoles en Twitter, y detectar algunos factores que podrían estar influyendo en la elección de uno u otro tono. El trabajo propone una triangulación de técnicas big data (para el análisis de sentimiento) y técnicas cuantitativas (análisis estadísticos a través del modelo de regresión), considerando diferentes fuentes de datos, tanto primarias como secundarias.

En primer lugar se ha efectuado una extracción de mensajes recientes de Twitter, como base para el posterior análisis de sentimiento. La extracción se realizó en mayo de 2017, y se limitó a la descarga de una muestra con los últimos 100 tweets para las cuentas en Twitter de los municipios españoles con más de 50.000 habitantes que respondieron el cuestionario del proyecto \#SocialGovNet (12.200 tweets descargados en total). Este proyecto, financiado por la Fundación $B B V A$, está permitiendo analizar las dinámicas de adopción y uso de redes sociales en los municipios españoles. 
Para la extracción se empleó un programa en Python, creado colaborativamente desde los repositorios de la comunidad GitHub. Es posible consultar su código desde este enlace: https://gist.github.com/yanofsky/5436496

El código emplea las funciones de "tweepy" y "csv" para efectuar las solicitudes a la API pública de Twitter. Una vez descargados los tweets solicitados, el programa los almacena en un fichero .csv (que puede ser leído por software como Excel). La limitación de la descarga en tweets se incorporó como especificación debido a la capacidad del software de análisis.

La elección de la herramienta de análisis de sentimiento viene de la mano de Indico. Este servicio ofrece análisis de sentimientos de calidad, aunque limitados en la ratio de tweets a analizar y a un nivel de confianza cercano al $80 \%$. Se trata de un sistema machine-learning, enfocado en el reconocimiento de emociones (Arcila-Calderón; Barbosa-Caro; Cabezuelo-Lorenzo, 2016). Este tipo de software ofrece la ventaja de ser capaz de aprender a medida que se introduce contenido en su sistema (Bae; Lee, 2012). El sistema recibe constantemente entradas de contenido, y los procesa según el tono (negativo, positivo o neutral).

A pesar de un cierto escepticismo hacia estos sistemas, la cantidad de información que pueden procesar es mayor que la permitida por codificaciones manuales, evitando muchos de los errores derivados de la codificación humana. Aunque debemos ser conscientes de que estos programas son todavía incapaces de capturar todas las vicisitudes de los contextos (Murthy; Preto, 2014), los modelos de análisis de sentimiento basados en machine-learning son capaces de manejar con menos recursos y en menor tiempo, mayores volúmenes de datos debido a sus capacidades de procesamiento y de aprendizaje a largo plazo (Arcila-Calderón; Barbosa-Caro; Cabezuelo-Lorenzo, 2016).

Las Ilamadas al aplicativo de Indico también se realizaron mediante Python, importando su función y empleando las distintas funciones que pueden consultarse en la documentación.

https://indico.io/docs

El training set se halló en el propio soporte ofrecido por la plataforma, y la participación de los investigadores fue limitada, supervisando únicamente los tweets que se consideraban dudosos. El aprendizaje se realizó con los mismos tweets de los ayuntamientos a analizar. Para la distinción del tono, aunque no es posible indicar todos, pues no se dispone de acceso al algoritmo de Indico, se solicitaron como relevantes la presencia de emoticonos, hashtags, expresiones (de agradecimiento, de rechazo...), el uso excesivo de mayúsculas, la presencia de meros enlaces, el empleo de palabras con connotaciones positivas o negativas, las frases hechas y las llamadas a la acción, entre otros.

El resultado ofrecido por el sistema distingue tres tipos de tono:

- negativo: al que asigna un valor entre 0 y 0,4 ;

- neutral, entre 0,4 y el 0,6;

- positivo, que iría entre 0,6 y 1 .

El sistema lee las cadenas de texto introducidas y verifica las palabras que encuentra en ellas. También tiene en cuenta los signos y los emoticonos (Bae; Lee, 2012). A pesar de que estos análisis no son capaces de entender muchas de las expresiones más dependientes de contextos determinados (Zavattaro; French; Mohanty, 2015; Murthy; Preto, 2014), el hecho de que sean capaces de aprender, ofrece márgenes de confianza en los resultados cada vez más elevados.

El tono general encontrado en los análisis sobre perfiles de organizaciones públicas por los estudios más recientes ha sido el neutro

Los resultados obtenidos para cada ayuntamiento fueron convertidos en una nueva variable, que se introdujo como parte de la base de datos del proyecto \#SocialGovNet. Esta base de datos contiene información sobre 122 municipios de más de 50.000 habitantes en España, recopiladas gracias a un cuestionario específico enviado a los gestores de redes sociales digitales de estos ayuntamientos, basado en un total de 22 preguntas dedicadas exclusivamente a identificar diferentes dimensiones de la adopción de estas tecnologías en el sector público. Los casos por regiones se agruparon de la siguiente manera: Andalucía (25 casos), Aragón (2), Asturias (1), Baleares (1), Canarias (7), Cantabria (2), Castilla la Mancha (6), Castilla y León (9), Cataluña (19), Ceuta (1), Comunidad Valenciana (13), Comunidad de Madrid (18), Extremadura (3), Galicia (4), La Rioja (1), Melilla (1), Murcia (4), Navarra (1) y País Vasco (4).

En la misma base de datos se han introducido a su vez variables sociodemográficas de interés, descargadas desde las bases del Instituto Nacional de Estadística (INE) y Transparencia Internacional. A partir de estos datos, el trabajo emplea análisis estadístico-descriptivos, y empírico-explicativos (mediante un modelo de regresión logística).

La operacionalización de las variables se ha llevado a cabo de la siguiente manera:

En primer lugar la variable dependiente principal se configura como el tono general empleado en esos perfiles. Siguiendo las mediciones explicitadas en la metodología, operacionalizamos el tono general (sentimiento) en tres categorías, según su valor agregado general (media de todos los tweets analizados) sea negativo (valores entre 0 y 0,4 ), neutro (valores entre 0,4 y 0,6 ) y positivo (valores entre 0,6 y 1 ).

La primera variable independiente respondía al interés por la formación recibida por los gestores públicos del ayuntamiento. Esta variable se encontraba incorporada ya en el cuestionario de \#SocialGovNet bajo la pregunta:

P.12: ¿Reciben formación para el manejo de redes sociales por parte de su Ayuntamiento?

Las opciones de respuesta eran "sí, ha recibido formación" y "no, no ha recibido formación”. En nuestra recodificación posterior, se eliminan únicamente las opciones de no sabe/ no contesta.

La segunda se refería al departamento o departamentos encargados de la gestión de redes sociales en el ayuntamiento. 
Nuevamente, esta variable puede encontrarse en la base de datos \#SocialGovNet bajo la pregunta:

P.8: ¿Qué unidades/departamentos intervienen en la gestión de los perfiles?

Las opciones de respuesta a esta pregunta son: Comunicación, Participación, Gobierno abierto, Informática, Todos los departamentos, Cultura, Juventud, Turismo, Deportes, Bienestar social, Emergencias y Otros. Para facilitar el análisis, nuestra recodificación se ha centrado fundamentalmente en dos categorías: comunicación y gestión por varios/todos los departamentos.

La tercera corresponde al uso de planes de medios sociales para la estrategia de adopción y uso de redes sociales digitales en el ayuntamiento. Ésta también se extrajo íntegramente de \#SocialGovNet, bajo la pregunta:

P.4: Por favor, indique si se ha desarrollado alguna guía para el uso de redes sociales digitales en su administración.

Las opciones de respuesta incluían "sí", "no, todavía no está previsto", "no, pero su desarrollo está previsto en menos de un año", "no, pero su desarrollo está previsto en más de un año". A efectos prácticos la operacionalización de la variable se ha considerado como "sí, se dispone de planes" y "no, no se dispone de planes".

La cuarta hace referencia a la consideración de la cultura organizativa como un inhibidor en la adopción y uso de redes sociales digitales dentro del sector público. También se extrajo de \#SocialGovNet, bajo la pregunta:

P.15: Las cuestiones siguientes se han considerado como inhibidores del uso de las redes sociales digitales en las administraciones públicas o como potenciales riesgos.

Tratándose de una escala tipo Likert donde el valor "1", se ha considerado como mínima percepción como inhibidor, y el valor "5", como máxima percepción como inhibidor.

La quinta variable independiente es de tipo socio-demográfico: edad media de la población del municipio. Esta variable fue incorporada posteriormente a la base de datos \#SocialGovNet, y se extrajo del INE. Su operacionalización se realiza mediante la media de edad de dicha población, y se muestra en valores discretos.

La sexta, el tamaño del municipio, se extrajo también de la base del INE, y se operacionalizó posteriormente en dos categorías:

- municipios entre 50.000 y 100.000 habitantes;

- municipios con más de 100.000 habitantes.

\section{Resultados}

Esta sección muestra los resultados preliminares del análisis de datos, que deben entenderse en el marco de una investigación exploratoria y sobre una materia innovadora, presente en las revisiones bibliográficas más recientes como un nicho de investigación que tiene un importante margen de desarroIlo futuro (Medaglia; Zheng, 2017). Se presentan atendiendo las dos preguntas de investigación y las hipótesis.
Tabla 2. Descriptivos para el sentimiento general en los perfiles en Twitter de ayuntamientos españoles

\begin{tabular}{|l|c|c|}
\hline \multicolumn{1}{|c|}{ Tono } & Frecuencia & Porcentaje \\
\hline Positivo & 69 & 68,32 \\
\hline Neutral & 32 & 31,68 \\
\hline Total (N) & 101 & 100 \\
\hline Tono general (media) & 0,68370 (Positivo) & \\
\hline Mínimo & 0,45390 (Neutral) & \\
\hline Máximo & 0,88275 (Positivo) & \\
\hline
\end{tabular}

\section{1. ¿Cuál es el tono general empleado por los ayun- tamientos en su uso de redes sociales?}

La bibliografía más reciente ha encontrado que el tono con el que las administraciones públicas comunican a través de perfiles sociales activa diferentes tipos de respuesta en la ciudadanía. El tono general encontrado en el estudio de Zavattaro, French y Mohanty (2015) fue el neutral, relacionándolo con el ánimo de mera provisión de información a través de estos perfiles, y con niveles de institucionalización de redes sociales menos elevados a los esperados. De ahí que nuestra primera hipótesis se formulase como: H1. Los gestores de redes sociales de las organizaciones públicas emplearán un tono generalmente neutro, en línea con una estrategia de provisión de información institucional. Nuestros análisis se han intentado repetir en el ámbito español, obteniendo los resultados de la tabla 2.

El número de perfiles sociales de los ayuntamientos con un sentimiento general positivo (69) es considerablemente mayor que aquellos con un tono neutral (32). Este resultado choca frontalmente con lo esperado siguiendo la bibliografía previa (Zavattaro; French; Mohanty, 2015), donde el tono preponderante parecía neutral. De la misma forma, y siguiendo esos mismos estudios, se destaca también la ausencia de resultados negativos generales dentro del tono, manteniéndose los ayuntamientos en mínimos alejados de esos tonos negativos (mínimo en 0,45 ). El número de casos reducido en comparación al total de respuestas del cuestionario debe encontrarse en que no todos los ayuntamientos tienen presencia en Twitter, aunque sí en otras redes sociales, como Facebook.

Un tono general tan positivo resulta llamativo, especialmente cuando otras investigaciones anteriores han encontrado que los estadios de implementación de estas tecnologías sociales en los municipios españoles todavía resultan ciertamente incipientes (Criado; Rojas-Martín, 2015). Sin embargo debe entenderse también que el caso español se ha situado en un nivel de difusión de estas tecnologías sociales muy elevado durante los últimos años, estimándose que más del $80 \%$ de la población española es usuaria de redes sociales (CIS, 2016). Este hecho también podría estar ocurriendo, aunque de forma algo matizada, en las administraciones públicas.

Las organizaciones públicas parecen estar haciendo un esfuerzo real por aprovechar las posibilidades de las tecno- 
Tabla 3. Modelos de regresión logística para cada grupo de factores explorados

\begin{tabular}{|c|c|c|c|}
\hline & Modelo 1 & Modelo 2 & Modelo 3 \\
\hline Formación en redes (0-No,1-Sí) & 0,33519524 & 0,09125762 & 0,10794858 \\
\hline Unidades de gestión (0-Comunicación,1-Varios o todos los departamentos) & $-0,61610006$ & $-0,65188917$ & $-0,63038741$ \\
\hline Planes de medios sociales (0-Sí, 1-No) & & $-0,78389193$ & $-0,82461186$ \\
\hline Cultura organizativa & & 0,13817735 & 0,15484946 \\
\hline Edad (media población) & & & $-0,07301545$ \\
\hline Tamaño municipio (0-50k-100k, 1-<100k)) & & & $-0,30012318$ \\
\hline$(\mathrm{N})$ & 96 & 90 & 90 \\
\hline \multicolumn{4}{|l|}{${ }^{*} \mathrm{p}<0,05 ;{ }^{* *} \mathrm{p}<0,01 ;{ }^{* * *} \mathrm{p}<0,001$} \\
\hline
\end{tabular}

logías sociales en las que tienen perfiles abiertos. Esta idea sugiere que el tono general positivo $(0,68370)$ es una parte más de un proceso lento pero constante de difusión de la innovación, donde las agencias están comenzando a fijarse en los pioneros del sector, como early-adopters (Rogers, 2003), y van adoptando paulatinamente algunas de las características destacadas por esos pioneros. Esto indica también que los ayuntamientos españoles, siguiendo el marco de difusión de las innovaciones de Rogers (2003), han dejado de ser organizaciones rezagadas, y se están convirtiendo poco a poco en el tipo ideal de "mayoría inicial", caracterizado por un mayor emprendimiento y aprovechamiento de las innovaciones tecnológicas, sin llegar sin embargo al aprovechamiento total.

Las administraciones públicas están haciendo un esfuerzo real por aprovechar las potencialidades interactivas de las tecnologías sociales

\section{2. ¿Qué factores explicativos se encuentran detrás de la adopción de un tono u otro?}

La segunda pregunta de investigación se refiere a los posibles factores condicionantes del tono empleado por los ayuntamientos en sus perfiles sociales. Para extraer algunos, se ha seguido el marco analítico de medición del éxito del gobierno digital propuesto por Gil-García (2012), que considera tres dimensiones: factores organizativos, institucionales y ambientales.

Para someter a análisis nuestras variables se han elaborado varios modelos de regresión no lineal. Nos hemos decantado por la regresión logística, con el objetivo de verificar la probabilidad de que los municipios empleen un tono $u$ otro en función de los factores presentados previamente, analizándolos uno a uno por separado mientras el resto de variables se mantienen constantes. La tabla 3 muestra estos análisis.

Cada modelo se identifica con la presencia adicional de uno de los factores de éxito presentes en el marco de Gil-García (2012):

- Modelo 1: agrupa los factores relativos a la dimensión organizativa;

- Modelo 2: incorpora a los anteriores los de la dimensión institucional;

- Modelo 3: incorpora los de los condicionantes ambientales.
Manteniendo constantes los efectos del resto de variables, los modelos planteados indican un incremento en la probabilidad de pasar de un tono neutral a un tono positivo al recibir formación en redes sociales. De la misma forma y como cabría esperar, el no disponer de planes de medios sociales, manteniendo el resto de variables constantes, genera un descenso en la probabilidad de encontrar tono positivo en los perfiles explorados. Esos descensos se aprecian al pasar de una gestión de los perfiles desde los departamentos de comunicación a una gestión inter-departamental, y también, aunque en menor medida, al hablar sobre la edad media de la población, disminuyendo la probabilidad de encontrar ese tono positivo conforme envejece. A pesar de lo anterior y como se aprecia en la tabla 3, ninguna de las variables de los modelos presenta una relación estadísticamente significativa. Es posible que ello se deba en parte a que las relaciones en algunos casos no son lineales (por ejemplo, entre el tamaño del municipio o la edad media de la población).

Los modelos de análisis de sentimiento basados en machine-learning son capaces de manejar, con menos recursos y en menor tiempo, mayores volúmenes de datos

Comprobando una posible multicolinearidad, la tabla 4 muestra el test VIF (variance inflation factor) para nuestro modelo final. La principal preocupación para ello en relación a nuestro modelo podría ser que a medida que el grado de multicolinearidad crece, las estimaciones para los coeficien-

Tabla 4. Diagnóstico de multicolinearidad mediante VIF (variance inflation factor).

\begin{tabular}{|l|c|c|}
\cline { 2 - 3 } \multicolumn{1}{c|}{} & VIF & $\mathbf{1 / V I F}$ \\
\hline Formación en redes & 1,33 & 0,749238 \\
\hline Planes de medios sociales & 1,31 & 0,761646 \\
\hline Unidades de gestión & 1,19 & 0,842962 \\
\hline Cultura organizativa & 1,14 & 0,879592 \\
\hline Tamaño del municipio & 1,05 & 0,948391 \\
\hline Edad (media población) & 1,03 & 0,972730 \\
\hline Media VIF & 1,18 & \\
\hline
\end{tabular}


tes de nuestra regresión se hacen más inestables y sus errores aumentan. Sin embargo los resultados mostrados por la tabla 4 parecen estar bien. Los VIF de las variables aparecen como inferiores al valor estadístico considerado como preocupante (habitualmente, 10). Por otro lado, la tolerancia de ese diagnóstico (1/VIF) es superior en todos los casos a 0,1. No se perciben problemas de colinearidad.

\section{Conclusiones}

Este trabajo ha analizado el sentimiento (sentiment analysis) con el que los ayuntamientos de más de 50.000 habitantes en España se comunican a través de sus perfiles en la red social Twitter.

La bibliografía más reciente ha identificado una relación clara entre el tono con el que las organizaciones públicas emiten sus mensajes, y el fomento de la participación y la colaboración pública a través de redes sociales. Los resultados de esta investigación han mostrado que los municipios españoles de más de 50.000 habitantes se están esforzando por emplear tonos positivos en sus perfiles sociales, lo cual demuestra un impulso cada vez más importante en el uso de estas herramientas.

\section{Los municipios de más de 50.000 habitan- tes se están esforzando por emplear tonos positivos en sus perfiles sociales, lo cual demuestra un impulso cada vez más im- portante en el uso de estas herramientas}

Sin embargo, nuestro estudio no ha permitido validar todas las hipótesis derivadas del análisis de la bibliografía en torno a la influencia que ciertos factores organizativos, institucionales y ambientales tienen sobre el empleo de esos diferentes tonos de comunicación. Lo anterior no implica que se deban descartar tales hipótesis, sino que es necesario profundizar en su análisis y mantener el interés por el estudio del efecto que tienen tales variables, incluyendo la formación de los empleados públicos sobre el uso de redes sociales, la unidad administrativa desde la que se gestionan, la existencia de planes de medios sociales, la cultura organizativa o las variables socio-demográficas, como la edad media de la población y el tamaño del ayuntamiento, sobre el nivel de implantación de las redes sociales en las administraciones locales.

Desde una perspectiva teórica este artículo ha realizado una revisión y avance sobre las variables que pueden estar detrás de los diferentes estilos de comunicación de las administraciones locales en redes sociales. La aplicación del modelo EEGS (Gil-García, 2012), que aborda el éxito del uso de las tecnologías en las organizaciones públicas, se ha confirmado como un buen punto de partida. Su conexión con la práctica de la comunicación sugiere que es necesario atender determinados rasgos organizativos, institucionales y ambientales (sobre todo, los socio-demográficos) para alcanzar unas metas comunicativas determinadas. Dicho de otra manera, frente a modelos iniciales basados en el éxito individual del community manager, para tener éxito en las redes sociales en un estadio de mayor madurez de este tipo de tecnologías, sobre todo en la interacción con la ciudadanía, será necesario promover modelos más complejos, que atiendan factores mucho más diversos que afectan a las organizaciones públicas.

Un trabajo de este tipo no está exento de puntos de mejora. Uno de ellos nos lleva a la reflexión sobre la variable dependiente (el tono), y su medición a través de software machine-learning.

Por un lado, el nivel de confianza ofrecido por la herramienta, en torno al $80 \%$, no parece todavía suficiente para establecer relaciones estadísticamente significativas. Seguramente sea esta una de las limitaciones más relevantes de esta investigación exploratoria. Cabe sin embargo que estos sistemas mejoren, conforme los programas vayan adquiriendo mayores recursos, y el uso continuado para investigaciones y análisis lleve a un aprendizaje creciente. El tipo de análisis realizado supone un avance en la investigación empírica sobre información y comunicación en las administraciones públicas, que merecerá la pena mejorar y extender en el futuro.

Por otro lado, será necesario aumentar el número de tweets a descargar, y ampliar el horizonte temporal de exploración de cara a obtener datos masivos más precisos sobre la actividad de los municipios en estas comunidades digitales.

Nuestro estudio presenta además otras posibles líneas de desarrollo que esperamos solventar en investigaciones futuras. Aunque las dimensiones del marco EEGS se han estructurado en torno a algunas variables relevantes, el modelo final debe valerse de un repertorio más amplio. Una variable interesante como refuerzo a la dimensión ambiental puede ser el partido político en el gobierno de la corporación municipal, comprobando si hay relación entre los tonos positivos y una información de carácter más partidista que institucional (Rodríguez-Bolívar, 2015). Además convendría identificar y diferenciar al hablar de figuras de gestión en redes sociales entre los directores de comunicación, los social media managers y los community managers. Estas dos últimas figuras merecen especial atención en futuros trabajos dado su creciente relevancia en el sector público.

Este trabajo abre una línea de investigación en redes sociales para comunicar datos masivos en las administraciones públicas. Por un lado, se inscribe en la línea de estudios destinada a la gestión y comprensión del comportamiento de la ciudadanía en su interacción con las organizaciones públicas a través de tecnologías sociales (Medaglia; Zheng, 2017). Por otro lado, y de forma más específica, participa de la línea innovadora de medir los sentimientos expresados a través de las nuevas tecnologías por parte de las organizaciones en sus procesos de comunicación (Zavattaro; French; Mohanty, 2015). Lo anterior es esencial y requerirá futuras aproximaciones, no sólo por la importancia de las emociones tanto en la sociedad como en la política, sino también por la necesidad de medir e identificar diferentes patrones comunicativos en las administraciones públicas dentro del nuevo escenario que ofrecen las redes sociales. 


\section{Nota sobre financiación}

Esta investigación es resultado del proyecto de investigación Gobernanza inteligente de las redes sociales digitales en las administraciones locales de la Unión Europea. Estrategias de implementación, factores de éxito y modelos de servicio público en España, Países Bajos, Reino Unido y Suecia" \#SocialGovNet (IN[16]_CJS_CCP_0190), financiado por la Fundación BBVA.

\section{Referencias}

Arcila-Calderón, Carlos; Barbosa-Caro, Eduar; Cabezuelo-Lorenzo, Francisco (2016). “Técnicas big data. Análisis de textos a gran escala para la investigación científica y periodística". El profesional de la información, v. 25, n. 4, pp. 623-631.

https://doi.org/10.3145/epi.2016.jul.12

Bae, Younggue; Lee, Hongchul (2012). "Sentiment analysis of Twitter audiences. Measuring the positive or negative influence of popular Twitterers". Journal of the American Society for Information Science and Technology, v. 63, n. 12, pp. 2521-2535.

https://doi.org/10.1002/asi.22768

Bailey, Claire; Singleton, Rico (2010). Friends, followers and feeds: National survey of social media use in state government. Lexington: Nascio.

https://goo.gl/BLQoik

Bertot, John C.; Jaeger, Paul T.; Grimes, Justin M. (2010). "Using ICTs to create a culture of transparency: E-government and social media as openness and anti-corruption tools for societies". Government information quarterly, v. 27, n. 3, pp. 264-271.

https://doi.org/10.1016/j.giq.2010.03.001

Bryer, Thomas (2013). "Designing social media strategies for effective citizen engagement: A case example and a model". National civic review, v. 102, n. 1, pp. 43-50.

https://doi.org/10.1002/ncr.21114

Chen, Qiang; Xu, Xialin; Cao, Bolin; Zhang, Wei (2016). "Social media policies as responses for social media affordances: The case of China". Government information quarterly, v. 33, n. 2, pp. 313-324.

https://doi.org/10.1016/j.giq.2016.04.008

CIS (2016). Barómetro de febrero de 2016. Estudio 3128. Madrid: Centro de Investigaciones Sociológicas.

http://www.cis.es/cis/export/sites/default/-Archivos/ Marginales/3120_3139/3128/es3128mar.pdf

Criado, J. Ignacio; Rojas-Martín, Francisco (eds.) (2013). Las redes sociales digitales en la gestión y las políticas públicas. Avances y desafíos para un gobierno abierto. Barcelona: Escola d'Administració Pública de Catalunya. ISBN: 97884695 93974

http://americo.usal.es/iberoame/sites/default/files/criado_ redes_sociales_digitales.pdf

Criado, J. Ignacio; Rojas-Martín, Francisco (2015). “Adopting social media in the local level of government: Towards a public administration 2.0?". En: Sobaci, Mehmet-Zahid (ed.). Social media and local government. New York: Springer, pp.
135-152. ISBN: 9783319177212

https://doi.org/10.1007/978-3-319-17722-9_8

Criado, J. Ignacio; Rojas-Martín, Francisco; Gil-García, J. Ramón (2017). "Enacting social media success in local public administrations: An empirical analysis of organizational, institutional, and contextual factors". International journal of public sector management, v. 30, n. 1, pp. 31-47.

https://doi.org/10.1108/IJPSM-03-2016-0053

Criado, J. Ignacio; Sandoval-Almazán, Rodrigo; Gil-García, J. Ramón (2013). "Government innovation through social media". Government information quarterly, v. 30, n. 4, pp. 319-326.

https://doi.org/10.1016/j.giq.2013.10.003

Criado, J. Ignacio; Villodre, Julián (2018). “Public employees in social media communities: Exploring factors for internal collaboration using social network analysis". First Monday, v. 23, n. 4.

https://doi.org/10.5210/fm.v23i4.8348

Eom, Seok-Jin; Hwang, Hanchan; Kim, Jun-Houng (2017). "Can social media increase government responsiveness? A case study of Seoul, Korea". Government information quarterly, v. 35, n. 1, pp. 109-122.

https://doi.org/10.1016/j.giq.2017.10.002

Fernández-Falero, María-Rosario; Trabadela-Robles, Javier; Garcés-Botacio, Indhira; Ruano-López, Soledad (2017). "Comunicación política de los ayuntamientos a través de sus webs. Caso de Extremadura". El profesional de la información, v. 26, n. 3, pp. 404-410.

https://doi.org/10.3145/epi.2017.may.06

Fountain, Jane (2001). Building the virtual state: Information technology and institutional change. Washington, D. C.: Brookings Institution Press. ISBN: 9780815700777

Gil-García, Juan-Ramón (2012). Enacting electronic government success: An integrative study of government-wide websites, organizational capabilities, and institutions. New York: Springer. ISBN: 9781461420149

Hofmann, Sara; Beverungen, Daniel; Räckers, Michael; Becker, Jörg (2013). "What makes local governments' online communications successful? Insights from a multi-method analysis of Facebook", Government information quarterly, v. 30, n. 4, pp. 387-396.

https://doi.org/10.1016/j.giq.2013.05.013

Lev-On, Azi; Steinfeld, Nili (2015). “Local engagement online: Municipal Facebook pages as hubs of interaction". Government information quarterly, v. 32, n. 3, pp. 299-307. https://doi.org/10.1016/j.giq.2015.05.007

Loukis, Euripidis; Charalabidis, Yannis; Androutsopoulou, Aggeliki (2017). "Promoting open innovation in the public sector through social media monitoring". Government information quarterly, v. 34, n. 1, pp. 99-109.

https://doi.org/10.1016/j.giq.2016.09.004

Ma, Liang (2016). "What drives the adoption of social media applications by the public sector?: Evidence from local health department". International journal of public administration in the digital age, v. 3, n. 4, pp. 76-93. 
https://doi.org/10.4018/IJPADA.2016100106

Manfredi-Sánchez, Juan-Luis; Corcoy-Rius, Marta; Herranzde-la-Casa, José-María (2017). “¿'Noticias de alcance? El criterio periodístico en la publicación de noticias en las webs municipales españolas (2011-2016)". El profesional de la información, v. 26, n. 3, pp. 412-420.

https://doi.org/10.3145/epi.2017.may.07

Medaglia, Rony; Zheng, Lei (2017). “Mapping government social media research and moving it forward: A framework and a research agenda". Government information quarterly, v. 34, n. 3, pp. 496-510.

https://doi.org/10.1016/j.giq.2017.06.001

Mergel, Ines (2013). "A framework for interpreting social media interactions in the public sector". Government information quarterly, v. 30, n. 4, pp. 327-334.

https://doi.org/10.1016/j.giq.2013.05.015

Mergel, Ines (2016). "Social media institutionalization in the U.S. federal government". Government information quarterly, v. 33, n. 1, pp. 142-148.

https://doi.org/10.1016/j.giq.2015.09.002

Murthy, Dhiraj; Preto, Laura R. (2014). “Comparing print coverage and tweets in elections. A case study of the 20112012 U.S. Republican primaries". Social science computer review, v. 33, n. 3, pp. 298-314.

https://doi.org/10.1177/0894439314541925
OASI (2016). La administración electrónica en Aragón - 2016. Observatorio Aragonés de la Sociedad de la Información. https://goo.gl/MVvEEQ

Rodríguez-Bolívar, Manuel-Pedro (2015). "Governance models for the delivery of public services through the Web 2.0 technologies: A political view in large Spanish municipalities". Social science computer review, v. 35, n. 2, pp. 203-225.

https://doi.org/10.1177/0894439315609919

Rogers, Everett M. (2003). Diffusion of innovations. New York: Free Press. ISBN: 9780743222099

Sixto-García, José (2012). "Uso de las redes sociales en la Administración pública gallega: ¿una técnica de marketing 2.0?". Pensar la publicidad, v. 6, n. 2, pp. 345-363.

https://doi.org/10.5209/rev_PEPU.2012.v6.n2.41220

Tello, Edgar (2008). "Las tecnologías de la información y comunicaciones (TIC) y la brecha digital: su impacto en la sociedad de México". Revista de universidad y sociedad del conocimiento, v. 4, n. 2, pp. 1-8.

https://doi.org/10.7238/rusc.v4i2.305

Zavattaro, Staci; French, Edward; Mohanty, Somya (2015). "A sentiment analysis of U.S. local government tweets: The connection between tone and citizen involvement". Government information quarterly, v. 32, n. 3, pp. 333-341. https://doi.org/10.1016/j.giq.2015.03.003
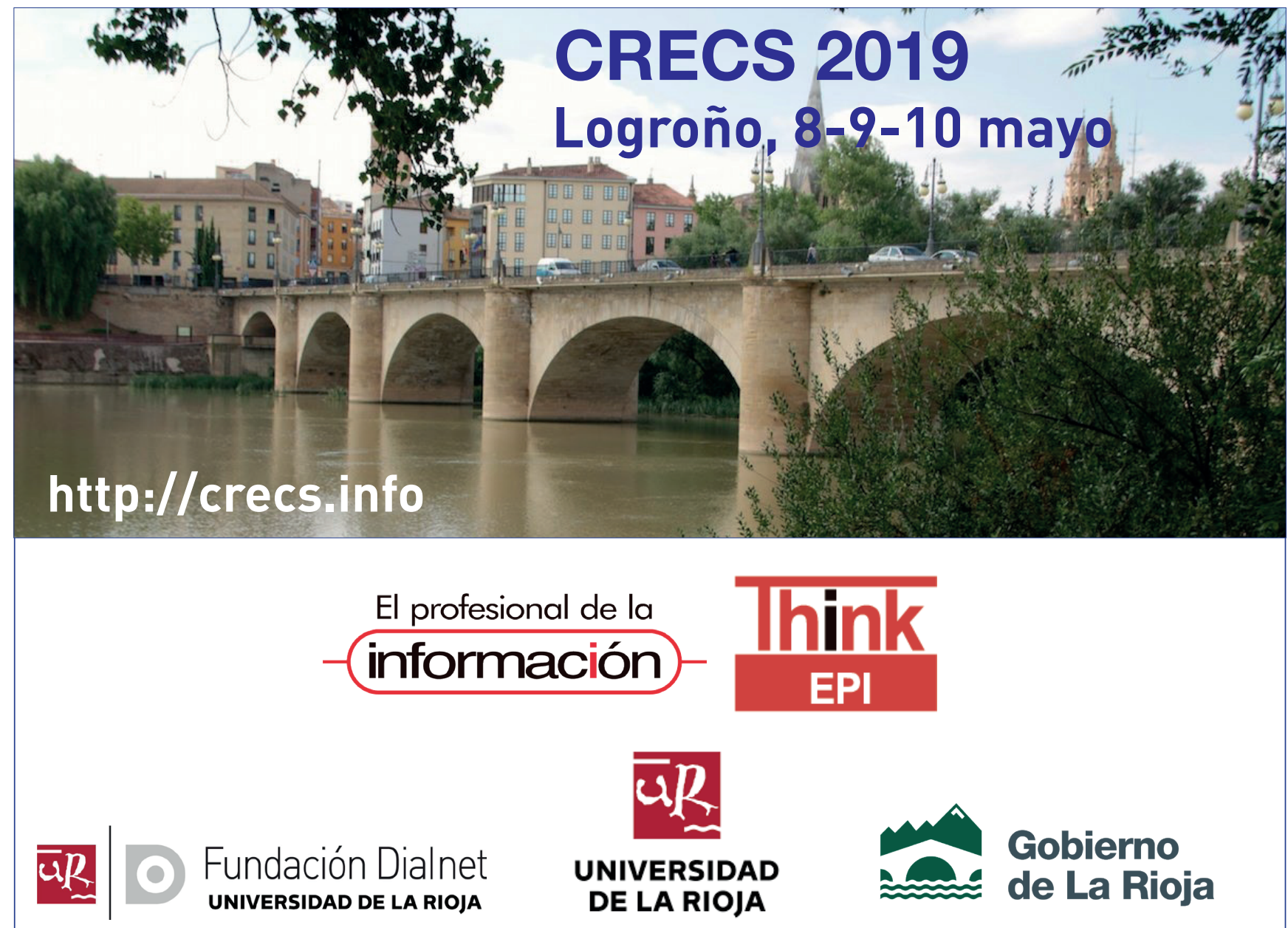